\title{
Lysimétrie en sol de craie non remanié. I. - Drainage, évaporation et rôle du couvert végétal. Résultats 1973- 1980
}

Jean-Louis BALLIF \& Pierre DUTIL

avec la collaboration technique de Daniel GOBERT \& Christian HERRE

I.N.R.A., Station d'Agronomie et de Science du Sol, Route de Montmirail, Fagnières F51000 Châlons/Marne

RÉSUMÉ

\begin{abstract}
Les drainages sont mesurés depuis 1973 sur des lysimètres, de $2 \mathrm{~m}$. de profondeur, en sol de craic non remanié. Pour les lysimètres cultivés, le drainage normal commence en décembre ct dure 4 à 5 mois. Leur drainage total annuel moyen $(160 \mathrm{~mm})$ représente 61 p. 100 des pluies d'hiver et 24 p. 100 des précipitations annuelles. Il est plus important en sol nu $(284 \mathrm{~mm})$ qu'en sol gazonné $(172 \mathrm{~mm})$. Sa durée et son volume dépendent des conditions climatiques et du mode d'occupation du sol. En période de drainage, le temps

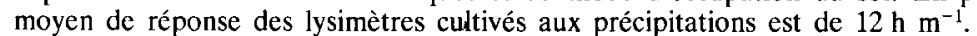

L'évaporation (pluie-drainage) varie selon le mode d'exploitation du sol. En lysimètre nu, l'évaporation est de $371 \mathrm{~mm}$; en lysimètre cultivé (betterave-blé), l'évapotranspiration est de $495 \mathrm{~mm}$. Le coefficient de transpiration serait de 4281 .
\end{abstract}

Mots clés additionnels : Lysimètres, sol nu, sols cultivés, gazon, betterave, blé, transpiration.

Lysimeter studies in undisturbed chalky soil. I. - Drainage, evaporation and role of plant cover. Results 1973-1980.

Drainage from undisturbed chalky soil has been measured since 1977 with lysimeters $2 \mathrm{~m}$. in depth. Under a crop cover, normal drainage began in December and lasted. Total mean annual drainage $(160 \mathrm{~mm})$ represented $61 \%$ of winter rain and $24 \%$ of total annual rain. For grazed soil, drainage was $172 \mathrm{~mm}$ and for bare soil $284 \mathrm{~mm}$. The duration and volume of drainage depended on climate conditions and on soil use. During the drainage period the mean response time of the lysimeters under crop cover was $12 \mathrm{~h} . \mathrm{m}^{-1}$. Evaporation (rain - drainage) varied according to the type of soil use. For bare soil, it was $371 \mathrm{~mm}$, while for soil under crop cover (sugar beet-wheat); it was $495 \mathrm{~mm}$. The transpiration coefficient was $428 \mathrm{l}$.

Key words : Lysimeter, bare soil, soil under crop cover, grazed soil, sugar beet, wheat, transpiration.

\section{INTRODUCTION}

La Champagne crayeuse représente une unité géologique et géomorphologique de 700000 ha environ. Les sols développés sur la craie sénonienne sont constitués en majeure partie par une rendzine brune développée sur un paléosol cryoturbé, formé àu cours des dernières périodes froides (DUTIL \& BALLIF, 1968). Ce profil pédologique est très complexe et l'étude de son fonctionnement saisonnier au point de vue hydrique, d'une part, et géochimique, d'autre part, a posé un problème de base au point de vue conception.

En définitive, le choix de cette conception s'est porté vers la construction de lysimètres avec sols en place, non

\footnotetext{
Revue d'Agronomic n' 9 -1983
}

remaniés. Actuellement, le dispositif d'étude comprend 12 lysimètres, qui fonctionnent depuis 1973. Il permet d'apprécier les transferts de l'eau entre 0 et $2 \mathrm{~m}$. de profondeur, sous le double aspect : quantitatif et qualitatif.

Les données recueillies à ce jour correspondant à des années climatiques suffisamment diversifiées, il apparaît nécessaire de commencer à publier des résultats de portée générale.

Cette première synthèse est consacrée aux caractéristiques essentielles du régime de l'eau à travers le sol, dans l'interaction «climat-sol-plante ». Ceci conduit à présenter : les relations : précipitations - drainage,

- l'évaporation,

- une interprétation du fonctionnement hydrique du sol en relation avec le mode d'occupation. 


\section{CONDITIONS EXPÉRIMENTALES}

Les lysimètres sont implantés dans les champs expérimentaux de la station I.N.R.A. et le sol des lysimètres est bien constitué par le profil type de la Champagne crayeuse. Une analyse très détaillée a été effectuée pour les études géochimiques (DURAND, 1978) et le dispositif a déjà été présenté (DuTIL, 1981). On rappellera simplement les caractères essentiels relatifs à son originalité.

Chaque lysimètre représente un cube de sol de $2 \mathrm{~m}$. d'arête, entouré de parois imperméables. Le sol ainsi isolé comprend, de haut en bas, les horizons suivants :

- l'horizon de surface $(0-25 \mathrm{~cm})$,

- les poches de cryoturbation ( 75 à $100 \mathrm{~cm}$ ), avec des cheminées d'ascension de la craie entre les poches,

- la craie remaniée $(20$ à $30 \mathrm{~cm})$,

- la craie en place fissurée (environ $50 \mathrm{~cm}$ ).

$\mathrm{Ce}$ monolithe repose sur 2 demi-plaques d'acier inoxydable préperforées, recouvrant 2 bacs qui recueillent les eaux de drainage. Dix lysimètres sont ainsi pourvus d'un plancher drainant à $2 \mathrm{~m}$. de profondeur ; un lysimètre a été coupé à $1,50 \mathrm{~m}$. de profondeur (lysimètre $\mathrm{n}^{\circ} 5$ ); enfin, une case de végétation est laissée en continuité avec le substrat géologique, par absence de plaque drainante.

A proximité immédiate se trouve le poste météorologique; les observations relatives aux conditions thermohydriques de l'ensemble du dispositif sont effectuées quotidiennement.

Ces lysimètres sont conduits selon 3 modes d'exploitation :

- sans fertilisation minérale : 2 cases (4 et 6 ), réservées aux études de géochimie en milieu crayeux ne reçoivent que les apports des pluies. Sur l'une, le sol est nu et sur l'autre le sol est engazonné ;

- avec fertilisation minérale: 4 cases en culture reçoivent des apports courants d'engrais et de pesticides. Elles répondent aux problèmes agronomiques régionaux. La rotation des cultures a été essentiellement betterave-blé, laissant un espace intercultural important (8 mois) entre le blé et la betterave. Une case porte 4 pieds de vigne « Pinot noir/41 B »;

- avec apports d'eaux résiduaires: 5 cases, cultivées avec la même rotation (betterave-blé), sont uniquement fertilisées avec des apports d'eaux résiduaires d'industries agricoles (féculerie - sucrerie). Elles permettent d'estimer le pouvoir épurateur du sol (DUTIL \& MULLER, 1979).

\section{RAPPELS DES CARACTÉRISTIQUES PHYSIQUES DU SOL POUR SES RELATIONS AVEC LA CIRCULATION DE L'EAU}

\section{A. Propriétés porales du sol}

Selon la défirition des horizons pédologiques déjà indiquée, les propriétés porales sont les suivantes :

- en surface $(0-25 \mathrm{~cm})$, l'horizon cultivé a une très bonne porosité totale, de 55 à $60 \mathrm{~cm}^{3} / 100 \mathrm{~cm}^{3}$,

- les horizons cryoturbés ont une porosité plus faible (41 à $55 \mathrm{p} .100$ ) et variable entre poches et cheminées d'ascension de la craie entre les poches, le centre des poches ayant parfois une faible porosité totale (34 à 39 p. 100),

- la craie remaniée, sous-jacente aux poches, a une porosité totale de $49 \mathrm{p} .100$,

- enfin, la craic en place, fissurée entre 150 à $200 \mathrm{~cm}$ de profondeur, a une porosité totale de 50 à 54 p. 100 ; celle des fragments de craie est plus faible ( 36 à 44 p. 100), la porosité de fissures de la craie variant de 3 à $10 \mathrm{p} .100$ (BALLIF, 1978).

La porosité des fragments de craie, à géométrie fixe, est constituée de pores ouverts de 0,3 à $3 \mu$ de diamètre. Ces pores sont classés dans une gamme réduite : $80 \mathrm{p} .100$ des vides ont un diamètre distribué entre 0,5 et $3 \mu$. C'est grâce à cette distribution que la craie retient l'eau et reste saturée.

Une autre conséquence de ce regroupement concerne la remontée capillaire (BALLIF \& VACHIER, 1981). C'est ce qui a été maintenu dans la case de végétation, case sans fond drainant. Par contre, dans les lysimètres, la remontée capillaire des horizons profonds de la craie a été interrompue et reste limitée à 1,5 ou $2 \mathrm{~m}$. d'épaisseur.

Ainsi dans chaque horizon des lysimètres, la porosité et la répartition des pores varient et, par conséquent, la perméabilité et les mouvements de l'eau diffèrent.

\section{B. Caractéristiques hydriques}

Les sols sur craie ont une très bonne réserve hydrique en profondeur : $560 \mathrm{~mm}$ de réserve utile sur $2 \mathrm{~m}$. d'épaisseur de sol (BALLIF, 1980). Ils se ressuyent rapidement et se dessèchent en surface. En période de sécheresse, la craie, à $150 \mathrm{~cm}$ de profondeur, reste encore saturée à $53 \mathrm{p} .100$ de sa porosité totale (BALLIF, 1981). Cette réserve est réalimentée par l'eau des horizons de craie sous-jacents.

En surface, la capacité au champ est de 21 p. 100 ; l'eau occupe alors 40 à 50 p. 100 de la porosité ou 25 p. $100 \mathrm{du}$ volume global du sol. La teneur en eau au point de flétrissement est faible $(10,6$ à 11,5 p. 100). Ces faibles humidités sont rencontrées pendant les périodes sèches.

En profondeur :

Dans les horizons cryoturbés, la capacité au champ varie de 17,5 à 17,8 p. 100 à l'intérieur des poches, alors que dans la craie remaniée elle est de 25,7 p. 100 et l'humidité au point de flétrissement est très faible (2 à 5 p. 100).

Dans la craie en place, la teneur en eau à la capacité au champ est de 25 à 26,9 p. 100 , ce qui correspond à un remplissage de 67 à $84 \mathrm{p}$. 100 de la porosité totale, soit 33 à 39 p. $100 \mathrm{du}$ volume global du sol. La teneur en eau au point de flétrissement est excessivement faible (pF. 4,2 =1,2 à 1,7 p. 100).

En période sèche $(1967,1976)$, les mesures sur le terrain ont montré que l'humidité de la craie n'atteint pas les faibles valeurs du point de flétrissement ou du $\mathrm{pF} 4,2$; cette humidité est de 19 à 21 p. 100.

\section{TABLEAU 1}

Réserve hydrique du sol près des lysimètres. Water reserve of soil near lysimeters.

$$
\begin{aligned}
R . U . & =Z \times d a \times(H c c-H p F 4,2) \\
Z & =\text { profondeur du sol en dm. } \\
\text { où } & =\text { densité apparente } \mathrm{g} / \mathrm{cm}^{3} . \\
H c c= & \text { humidité à la capacité au champ g/l00 g. } \\
H p F 4,2 & =\text { humidité au point de flétrissement. } \\
& \text { R.F.U. observée }: Z \times \text { da } \times(H c c-H \text { mini }) .
\end{aligned}
$$

\begin{tabular}{ccc}
\hline Profondeur & $\begin{array}{c}\text { Réserve Utile } \\
\text { R.U. }\end{array}$ & $\begin{array}{c}\text { R.F. utilisée } \\
\text { observée }\end{array}$ \\
\hline $0-25$ & 35,4 & 33 \\
$0-50$ & 102,3 & 53,4 \\
$0-75$ & 169,2 & 73,8 \\
$0-100$ & 236,1 & 94,2 \\
$0-150$ & 391,4 & 139 \\
$0-200$ & 561,4 & 190 \\
\hline
\end{tabular}


Aussi pour calculer la réserve utilisée (RFU) observée du sol sur craie, nous remplaçons la limite du point de flétrissement par l'humidité minimale mesurée en période de sécheresse estivale (tabl. 1). La réserve facilement utilisable observée est de $190 \mathrm{~mm}$ sur $200 \mathrm{~cm}$ et de $95 \mathrm{~mm}$ sur $100 \mathrm{~cm}$ de profondeur.

\section{RÉSULTATS}

\section{A. Généralités sur les observations}

\section{L'année lysimétrique}

Pour la présentation des résultats, il a été adopté une année lysimétrique, qui commence le $1^{\text {er }}$ octobre et finit le 30 septembre de l'année suivante. Elle coïncide avec l'année agricole et une telle délimitation a l'avantage d'englober un cycle végétatif complet et une période complète de drainage dans l'année d'observations.

\section{Les conditions climatiques générales}

Pour la période considérée (1973-1980), il y a eu une année très sèche (1975-76) et 2 années humides (1977-78) et (1979-80).

La normale pour les précipitations est de $633 \mathrm{~mm} / \mathrm{an}$, pour une période de 30 ans. La température moyenne annuelle sous abri est de $9,9^{\circ} \mathrm{C}$.

\section{Le drainage annuel}

Sur un cycle annuel, le fonctionnement hydrique des lysimètres permet de définir 2 périodes :

— une période de drainage, qui se situe pendant tout l'hiver, au cours de laquelle le drainage est égal ou supérieur à $1 \mathrm{~mm}$ par décade ; cette dernière valeur a été retenue car elle correspond à un changement rapide de la concentration des eaux en éléments minéraux (BALLIF \& MULLER, 1981);

- une période de non-drainage, qui couvre le reste de l'année.

\section{B. Les relations « Précipitations-Drainage »}

\section{Durée du drainage (fig. 1)}

- Les lysimètres cultivés commencent à drainer normalement en décembre et le drainage s'arrête entre la mi-avril et la mi-mai. Ainsi, en sols cultivés, le drainage dure 4 à 5 mois.

A noter que pour le lysimètre 5 (de $1,50 \mathrm{~m}$. de profondeur), avec les mêmes cultures, le drainage commence et s'arrête avant celui du lysimètre 3 (de $2 \mathrm{~m}$. de profondeur). Au cours de l'année sèche (1975-76), le drainage a duré très peu de temps (1 mois) et le lysimètre 5 n'a pas drainé.

- En sol $n u$, la reprise du drainage est avancée d'un mois et l'arrêt est plus tardif (mai à juillet); des reprises de courte durée sont fréquentes au cours du printemps et de l'été, selon l'importance des précipitations. Ainsi, en sol nu, le drainage dure 7 mois environ.

- En sol engazonné, 4 années après l'implantation, la durée du drainage devient légèrement supérieure à celle des sols cultivés.

\section{Volume d'eau drainé (tabl. 2)}

Dans cette publication, on ne peut reproduire les résultats décadaires obtenus pendant 7 ans sur chaque case. La présentation est limitée aux résultats annuels pour chaque case sur les plans de mesures. Très simplement, on peut

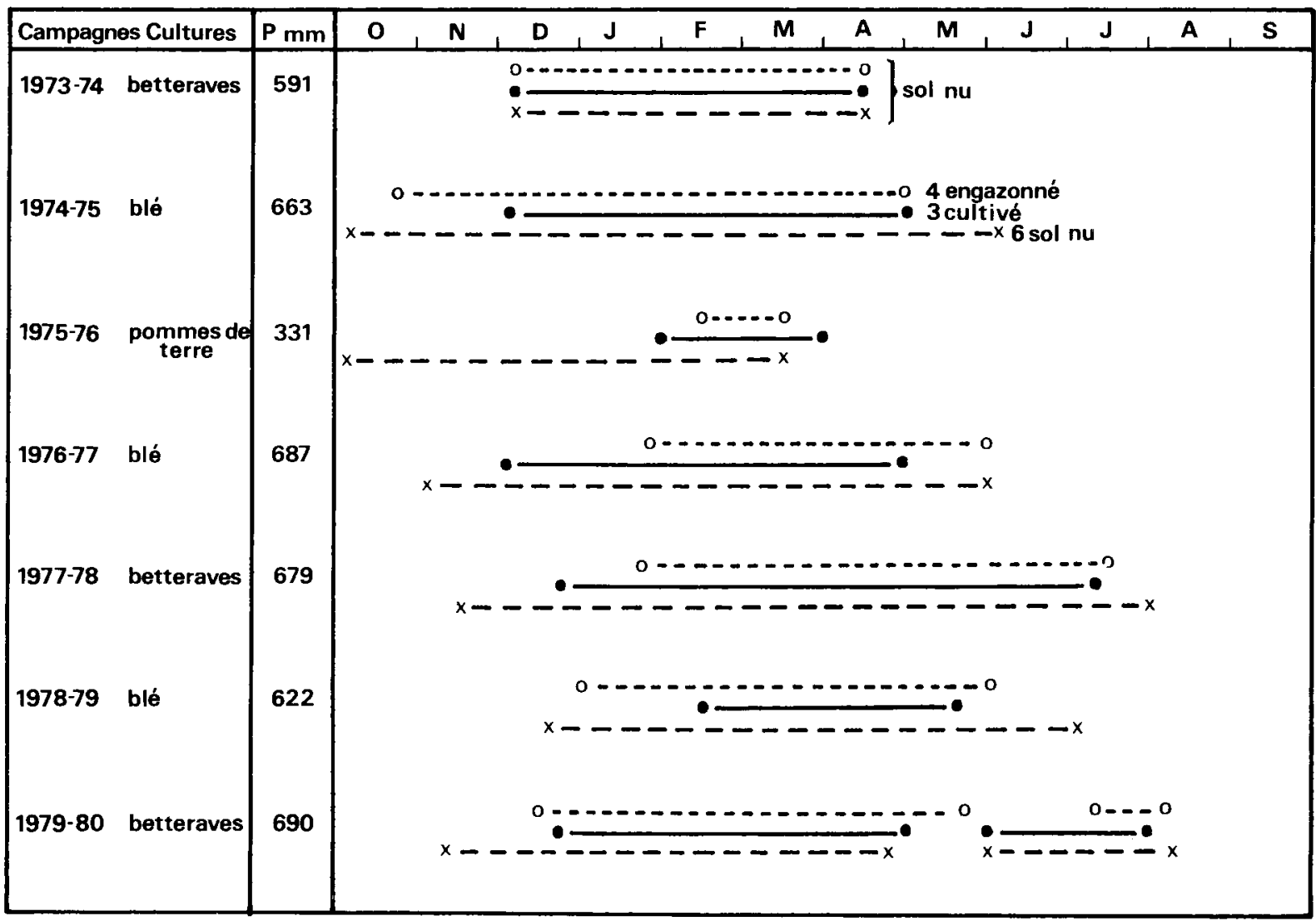

Figure 1

Durée du drainage des lysimètres 3-4 et 6 .

Drainage time of lysimeters 3,4 and 6.

\section{Lysimètre}

3 cultivé - Cropped

4 engazonné - grazed

6 sans végétation - bare soil 
TABLEAU 2

Drainages annuels des lysimètres. Moyenne des sept premières années (à l'exception de 1975-1976). Annual drainages of lysimeters. Average of first seven years (except 1975-1976).

\begin{tabular}{|c|c|c|c|c|c|c|c|c|c|c|c|c|c|}
\hline \multirow[b]{2}{*}{ Années } & \multirow[b]{2}{*}{ Cultures } & \multirow{2}{*}{$\begin{array}{c}\text { Pluie } \\
\text { totale } \\
\mathrm{mm}\end{array}$} & \multicolumn{11}{|c|}{ Drainage (en mm) } \\
\hline & & & $\begin{array}{c}1 \\
\text { Cultivé }\left({ }^{+}\right)\end{array}$ & $\stackrel{2}{2}$ & $\stackrel{3}{\text { Cultivé }}$ & $\begin{array}{c}4 \\
\text { Gazon }\end{array}$ & $\begin{array}{c}5 \\
\text { Cultivé }\end{array}$ & $\begin{array}{c}6 \\
\text { Sol nu }\end{array}$ & $\begin{array}{c}8 \\
\text { Cultivé }\left({ }^{+}\right)\end{array}$ & $\begin{array}{c}9 \\
\text { Cultivé }\left({ }^{+}\right)\end{array}$ & $\begin{array}{c}10 \\
\text { Cultivé }\left(^{+}\right)\end{array}$ & $\begin{array}{c}11 \\
\text { Cultivé }\left(^{+}\right)\end{array}$ & $\begin{array}{c}12 \\
\text { Cultivé }\end{array}$ \\
\hline $1973-1974$ & betterave & 591 & $242,8\left(^{+}\right)$ & 86,5 & 114,2 & 114,6 & 153,1 & 133,1 & 163,7 & & & & \\
\hline $1974-1975$ & blé & 663 & 110,4 & 224,4 & 135,4 & 199,3 & 143,0 & 301,9 & 146,7 & & & & \\
\hline $1975-1976$ & p. de terre & 331 & $71,6\left(^{+}\right)$ & 62,8 & 18,8 & 20,5 & 2,1 & 104,2 & 6,8 & & & & \\
\hline 1976-1977 & blé & 687 & 172,5 & 119,0 & 181,9 & 113,2 & 181,5 & 280,0 & 173,2 & 235,7 & 217,9 & 233,5 & 229,5 \\
\hline $1977-1978$ & betterave & $679\left(^{+}\right)$ & 365,0 & 277,7 & 225,5 & 151,4 & 240,1 & 351,0 & $596,4\left(^{+}\right)$ & $330,1\left(^{+}\right)$ & $335,7\left(^{+}\right)$ & $293,8\left(^{+}\right)$ & $358,3\left(^{+}\right)$ \\
\hline 1978-1979 & blé & 522 & 93,7 & 235,9 & 115,8 & 232,9 & 102,0 & 320,0 & 104,1 & 103,7 & 122,2 & 101,9 & 120,8 \\
\hline $1979-1980$ & betterave & 690 & 199,9 & 234,6 & 180,8 & 220,1 & 172,3 & 318,6 & 190,8 & 199,3 & 214,5 & $172,5\left(^{+}\right)$ & $251,1\left(^{+}\right)$ \\
\hline Moyenne & & 655 & 197,3 & 196,3 & 158,9 & 171,9 & 165,3 & 284,1 & $155,7\left(^{*}\right)$ & 212,1 & 222,5 & 200,4 & 239,9 \\
\hline $\mathrm{D} / \mathrm{P}$ en $\%$ & & & 30 & 30 & 23 & 26 & 25 & 42 & 24 & 31 & 33 & 29 & 35 \\
\hline P-D moyenne & & & 458 & 456 & 496 & 484 & 490 & 371 & 495 & 457 & 447 & 469 & 430 \\
\hline $\mathrm{D} / \mathrm{P}\left({ }^{*}\right)$ en $\%$ & & & 65 & 59 & 62 & 64 & 61 & 71 & 77 & 56 & 57 & 51 & 53 \\
\hline
\end{tabular}

$\left({ }^{*}\right)$ Case 8 - maïs en 1974 - moyenne, en éliminant 1977-1978, apport de $755 \mathrm{~mm}$ d'eau à raison de $20 \mathrm{ljj}$. du 18/10 au 18/04. Cultivé $\left({ }^{+}\right)=$avec apports d'eaux résiduaires.

(*) D/P de la période de drainage.

indiquer que le volume du drainage annuel reste étroitement lié à l'importance des précipitations.

En exemple, l'évolution des précipitations et du drainage est présentée pour 2 campagnes : 1978-79 et 1979-80 (fig. 2).

a) En 1978-79, après un automne particulièrement sec ( $P$ octobre-novembre $=37 \mathrm{~mm}$ ), le drainage des sols nus et engazonnés reprend en décembre et celui des sols cultivés en février seulement. Le volume total d'eau drainé est :

$$
\begin{array}{ll}
\text { — sol nu } & : 320 \mathrm{~mm} \\
\text { — sol engazonné } & : 232,9 \mathrm{~mm} \\
\text { - sol cultivé } & : 115,8 \mathrm{~mm}
\end{array}
$$

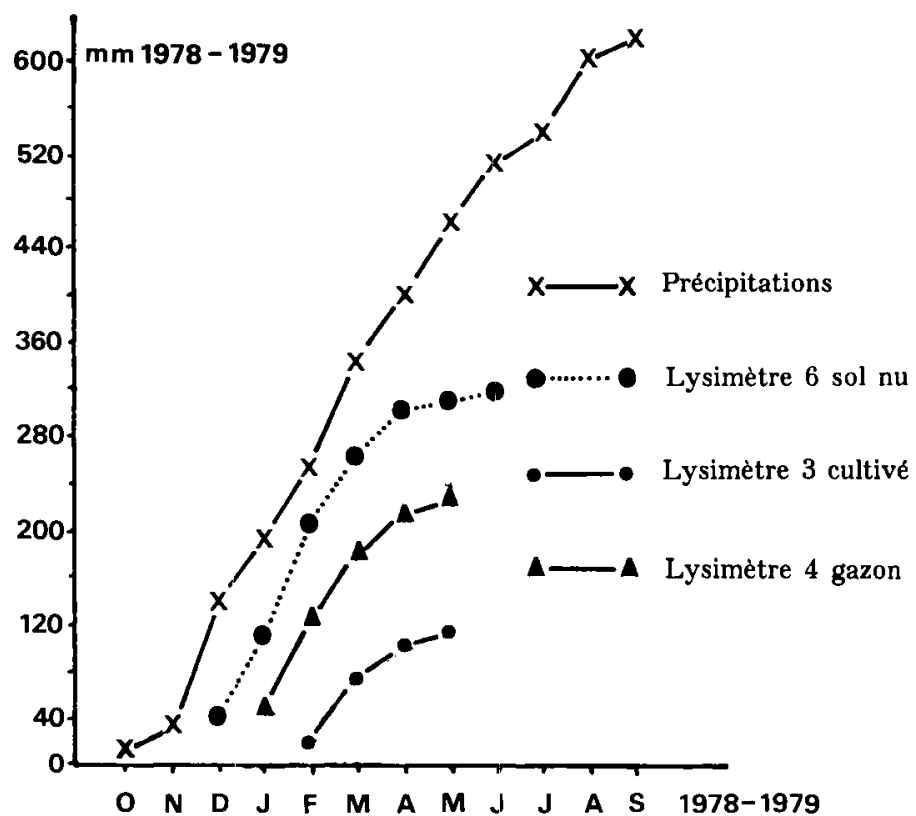

Figure 2

Evolution des précipitations et des drainages cumulés.

Accumulated rainfall and drainage. et pour la période du drainage hivernal, le drainage varie entre 51 et 53 p. 100 des précipitations.

b) En 1979-80, après un automne normalement pluvieux $(\mathrm{P}$ octobre-novembre $=117 \mathrm{~mm})$, la reprise du drainage s'effectue début novembre en sol nu et à la midécembre en sols cultivés et engazonnés. Les pluies de décembre sont importantes $(P=91 \mathrm{~mm})$. Les volumes drainés totaux sont les suivants :

$$
\begin{array}{ll}
\text { — sol nu } & : 318,6 \mathrm{~mm} \\
\text { — sol engazonné } & : 220,1 \mathrm{~mm} \\
\text { — sol cultivé } & : 180,8 \mathrm{~mm}
\end{array}
$$

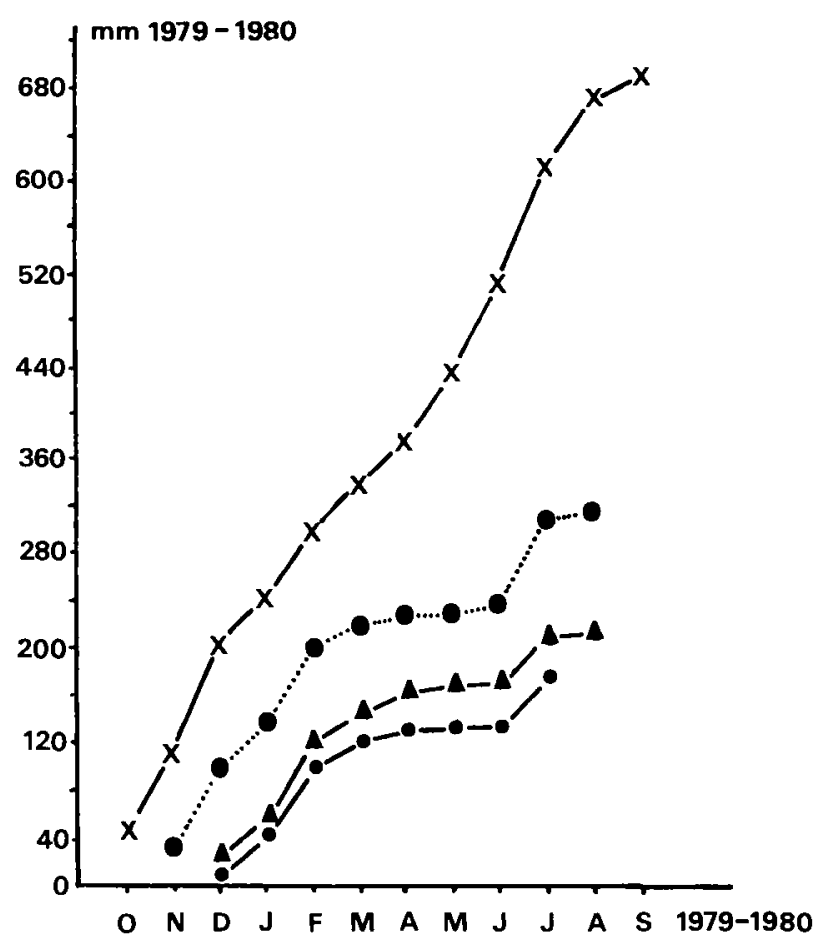


et, pour la période hivernale, le drainage représente 85 p. 100 des précipitations en sol nu, 70 p. 100 en sols cultivés et 76 p. 100 en sol engazonné.

De plus, on remarquera que des précipitations printanières et estivales importantes ( $P$ mai-juin-juillet $=177 \mathrm{~mm}$ ) peuvent provoquer une phase estivale de drainage d'une durée de 2 mois; les volumes supplémentaires drainés sont :

$$
\begin{array}{ll}
\text { — sol nu } & : 88 \mathrm{~mm} \\
\text { 一 sol engazonné } & : 53 \mathrm{~mm} \\
\text { — sol cultivé } & : 44 \mathrm{~mm}
\end{array}
$$

En l'état actuel de l'expérimentation, les valeurs moyennes que l'on peut fournir sont les suivantes:

- en sol cultivé, le drainage total annuel moyen est de $160 \mathrm{~mm}$, le drainage hivernal s'élevant à $25-30 \mathrm{~mm} / \mathrm{mois}$; ce drainage représente 24 p. 100 des précipitations annuelles;

- en sol gazonné, le drainage total annuel moyen est de $172 \mathrm{~mm}$, soit 26 p. 100 des précïpitations annuelles ;

- en sol nu, le drainage total annuel moyen est le plus élevé $(284 \mathrm{~mm})$, représentant 42 p. 100 des précipitations annuelles.

Enfin, on peut remarquer que le drainage hivernal moyen de la période stricte de drainage est de: 62 p. 100 en sol cultivé, 61 p. 100 en sol engazonné et 71 p. 100 en sol nu.

\section{Corrélations "Précipitations-Drainage"}

Les précipitations sont cumulées sur une base décadaire dans la période entre le moment ou P-ETP devient positif et la date d'arrêt du drainage.

a) Sous culture, avec une rotation betterave-blé, le drainage hivernal des lysimètres varie entre 133 et $225 \mathrm{~mm}$, soit 65 p. 100 des pluies hivernales. La corrélation entre les précipitations (P) et le drainage (D) est moyenne: $\mathrm{D}=0,493 \mathrm{P}-55,68$; coefficient de corrélation $\mathrm{r}=0,864$.

b) En sol $n u$, les drainages sont les plus importants (230 à $250 \mathrm{~mm}$ ). Ils varient entre 65 et 85 p. 100 des pluies. La corrélation entre les précipitations et le drainage est bonne : $\mathrm{D}=0,663 \mathrm{P}-33,85 ; \mathrm{r}=0,923$.

c) Sous gazon, le volume drainé varie (112 à $231 \mathrm{~mm})$.
La corrélation entre les précipitations et le drainage est mauvaise : $\mathrm{D}=\mathbf{0 , 3 6 1} \mathrm{P}+10,4 ; \mathrm{r}=0,578$.

En effet, en sol engazonné, l'herbe n'est pas coupée ; il s'est formé une épaisse couche d'herbe sèche. Les précipitations de faible intensité restent dans cette couche. La réhumectation du sol se trouve done limitée.

\section{Evaporation et évapotranspiration des lysimètres}

1. Avec un tel dispositif expérimental et son équipement actuel, on ne peut fournir des résultats que pour l'évaporation du sol nu et l'évapotranspiration de la case engazonnée.

L'estimation ne peut être faite qu'à partir de l'équation générale du bilan de l'eau des sols :

$$
\mathrm{P}=\mathrm{D}+\mathrm{E}+\triangle \mathrm{R}
$$

où $P$ représente les précipitations, $D$ le drainage, $E$ l'évaporation (ou l'évapotranspiration) et $\Delta \mathrm{R}$ la variation de la rétention d'eau dans une case, pour une période donnée.

Pour minimiser la valeur de $\Delta R$, les calculs ont été effectués sur 7 ans de mesures, entre 2 phases d'état hydrique similaires : sur cette période : E \# P - D.

- En sol nu, l'évaporation moyenne est de : $371 \mathrm{~mm} / \mathrm{an}$.

- En sol engazonné, l'évapotranspiration moyenne est de : $483 \mathrm{~mm} / \mathrm{an}$.

2. Pour les cases cultivées, avec une rotation betterave-blé, et en l'absence de mesures de l'état hydrique du sol en début et en fin de chaque cycle végétatif, on ne peut donner qu'une valeur globale pour le système cultural.

Ainsi, l'évapotranspiration moyenne annuelle du système betterave-blé, après 3 rotations est de $495 \mathrm{~mm}$.

En séparant les valeurs obtenues pour chaque année culturale de betterave ou de blé, les évapotranspirations moyennes sont respectivement de : $515 \mathrm{~mm}$ pour le blé et $476 \mathrm{~mm}$ pour la betterave. Rappelons cependant que ces valeurs ne sont qu'approximatives, en l'absence de possibilité de mesure de $\Delta \mathrm{R}$ sur le lysimètre pour chaque cycle végétatif..

3. Comparaisons avec d'autres installations lysimétriques (tabl. 3)

Les Stations françaises équipées de lysimètres sont réparties sous diverses conditions climatiques et pédologiques. Il

TABLEAU 3

Evaporation des lysimètres $(P-D)$ en $\mathrm{mm}$.

Evaporation of lysimeters.

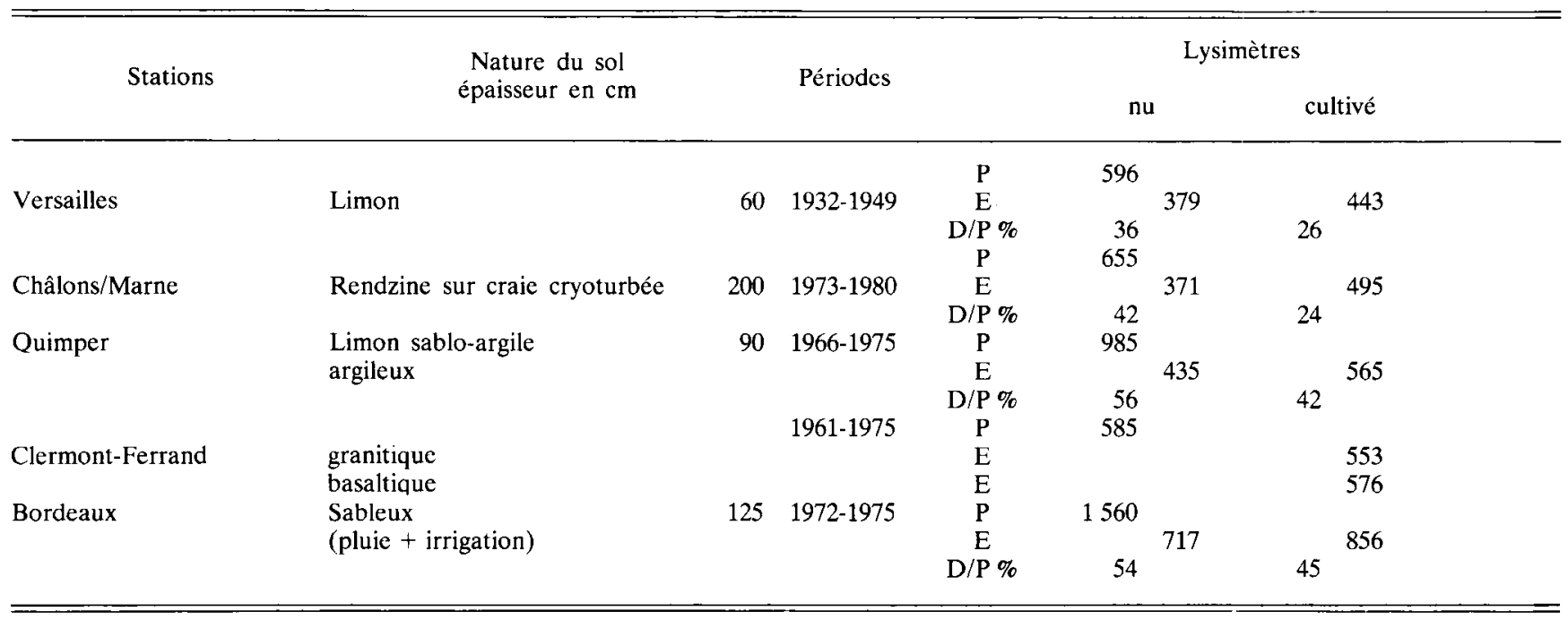



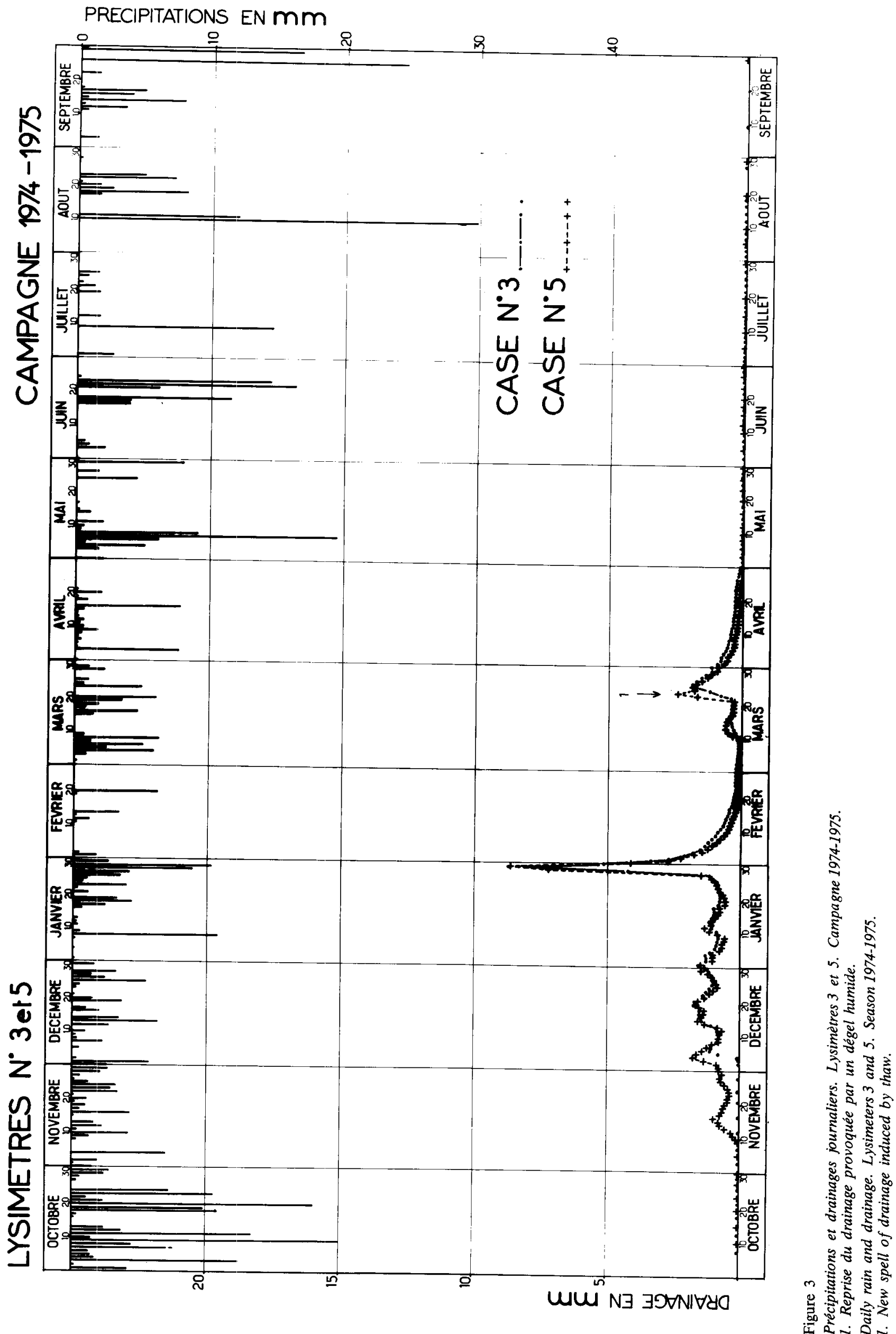

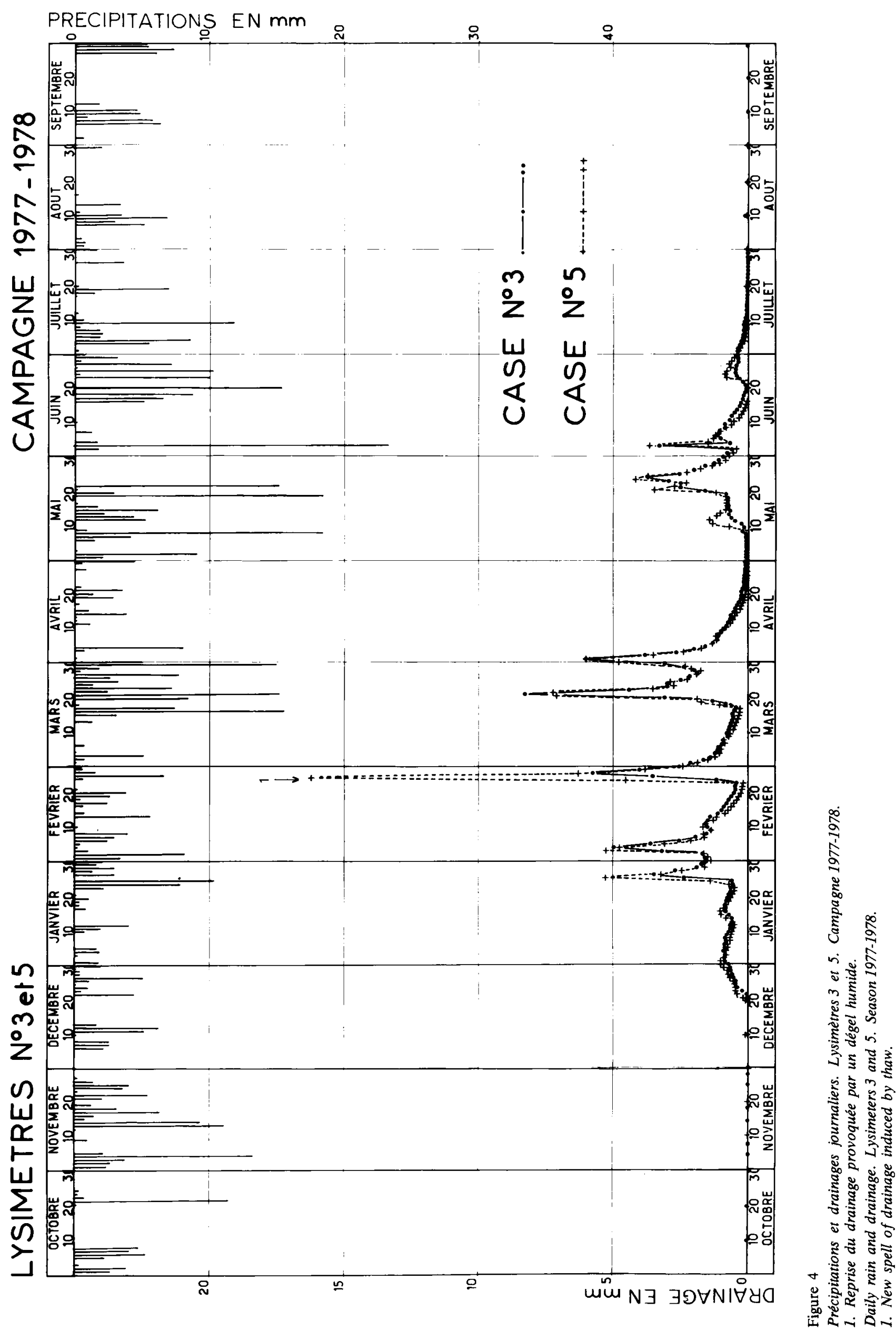
est toutefois intéressant de comparer les valeurs d'évaporation obtenues en sols nus et cultivés.

- En sols nus, l'évaporation varie assez peu selon les régions : 379 et $371 \mathrm{~mm}$ à Versailles et Châlons, et $435 \mathrm{~mm}$ à Quimper sous le climat de la côte atlantique. Le rapport drainage/précipitations varie de 36 à 56 p. 100 . Les valeurs élevées de Bordeaux (54 p. 100) et Quimper (56 p. 100) correspondant à des conditions de milieux particuliers (JUSTE et al., 1982 ; COPPENET, 1969).

- En sols cultivés, l'évaporation varie selon la nature des cultures pratiquées. Les valeurs sont comprises entre $443 \mathrm{~mm}$ à Versailles et $576 \mathrm{~mm}$ à Clermont-Ferrand, (DEJOU et MORIZET, 1977), Bordeaux $(856 \mathrm{~mm})$ se situant en conditions particulières (sols sableux et maïs avec irrigation).

\section{FONCTIONNEMENT HYDRIQUE DU SOL}

\section{A. Fonctionnement général du drainage hivernal : débits moyens et crues}

Au cours du drainage hivernal, l'écoulement s'effectue avec une intensité variable en fonction des pluies d'une manière générale et du gel d'une manière accidentelle. Une vue du déroulement du drainage est donnée en exemple pour 2 campagnes : $1974-75$ et $1977-78$ (fig. 3 et 4 ).

Les variations d'intensité et de volume de la pluie créent des cycles de crues et décrues successives du drainage. Ainsi, chaque hiver selon les conditions climatiques, on peut enregistrer 3 à 6 reprises de drainage important.

Le débit moyen journalier est de 0,5 à $1 \mathrm{~mm}$ et ce débit est identique pour tous les lysimètres.

Lors des reprises de drainage, le débit journalier de pointe est de 5 a $6 \mathrm{~mm}$, mais peut atteindre exceptionnellement 15 à $17 \mathrm{~mm}$ ( 2 fois sur 7 ans).

\section{B. Cas particulier du phénomène : gel-dégel}

Les plus forts débits de pointe sont enregistrés à la suite des dégels humides (mars 1975 ; février 1978 ; mars 1979 ; janvier 1980). Dans ces cas-là, les débits journaliers peuvent être multipliés par 5 à 12 , exceptionnellement par 40 à 60 (après période de gel et neige, février 1978).

Le mécanisme a déjà été indiqué (MAGNIANT \& DuTIL, 1971): lorsque le gel survient sur le sol en cours de drainage, le sous-sol se met en dépression et lorsque le dégel se produit, accompagné de pluies, la vitesse et le débit de drainage augmentent très fortement.

\section{Temps de réponse du drainage aux précipitations}

En période de drainage, les principaux faits marquants obtenus sont les suivants :

- Les faibles précipitations $\leqslant 10 \mathrm{~mm}$ ne fournissent pas de réponse au drainage à $2 \mathrm{~m}$. de profondeur.

- Des pluies de 13 à $15 \mathrm{~mm}$ cumulées sur 3 jours provoquent une reprise du drainage. Si cette pluie survient à la fin d'une période de précipitations, le drainage reprend en moins de $24 \mathrm{~h}$., avec un débit de 9 à $10 \mathrm{~mm} / \mathrm{j}$. Dans ce cas, le temps moyen de réponse du drainage aux précipitations est de $12 \mathrm{~h} . \mathrm{m}^{-1}$.

- A $150 \mathrm{~cm}$ de profondeur (lysimètre $\mathrm{n}^{\circ} 5$ ), les reprises du drainage sont en général en avance d'un jour sur celles des autres lysimètres. A ces reprises, le débit est plus intense que ceux des autres lysimètres, mais en décrue le débit est plus faible.

D. Quantités d'eau nécessaires pour la reprise du drainage en fonction du mode d'occupation du sol et du précédent cultural

La reprise de drainage d'un sol s'effectue lorsque ses différents horizons ont atteint une humidité supérieure à la capacité au champ. Pour le sol des lysimètres, de $2 \mathrm{~m}$. d'épaisseur, la réserve hydrique a été évaluée à $561 \mathrm{~mm}$ et la fraction utilisable à $190 \mathrm{~mm}$ (tabl. 1) ; cette dernière valeur correspondrait à une valeur maximum d'assèchement du sol après culture.

Par ailleurs, on a essayé de déterminer la quantité de pluie nécessaire, après l'été ou les récoltes, pour déclencher la reprise du drainage hivernal. Les meilleurs résultats ont été obtenus, non avec les valeurs de P, mais (P-ETP); l'ETP est calculée par la formule de TURC (1954).

On peut ainsi fournir quelques résultats en fonction du mode d'occupation du sol et par type de précédent cultural.

Tout d'abord, en sol $n u$, on constate que la reprise de drainage est plus précoce qu'en sol cultivé et il suffit de $40 \mathrm{~mm}$ (P-ETP) pour assurer la reprise de drainage.

En sol cultivé, cette reprise est tardive et varie en fonction du précédent cultural, c'est-à-dire de la date de récolte :

- Après blé, qui laisse le sol nu à partir de la mi-août, la reprise se produit après un cumul voisin de $100 \mathrm{~mm}(\mathrm{P}$ ETP) ;

- Par contre, après betterave, récoltée en octobre-novembre seulement, il y a eu un assèchement plus élevé et il faut environ $160 \mathrm{~mm}$ (P-ETP) pour assurer la reprise.

En définitive, c'est le mode d'occupation du sol et le type de précédent cultural, par le degré d'assèchement du sol qui a été provoqué, qui règlent la reprise du drainage hivernal, en relation avec l'intensité des pluies automnales.

Une exploitation de ces résultats est déjà utilisée pour la gestion raisonnée de l'épandage des eaux résiduaires des industries agro-alimentaires de la région (MULLER, 1977).

\section{E. Volume drainé et réserve hydrique du sol}

La réserve hydrique globale du sol, à la capacité au champ (hcc), mesurée au voisinage des lysimètres, est de $630 \mathrm{~mm}$ sur $2 \mathrm{~m}$. de profondeur. Le drainage annuel des cases lysimétriques cultivées représente donc 18 à 36 p. 100 de cette réserve hydrique; en case nue, ce pourcentage varie de 44 à 55 p. 100 .

En moyenne, le pourcentage du drainage par rapport à la réserve hydrique globale est :

- en case cultivée de 25 p. 100 ;

- en case nue de 45 p. 100 ;

- en case engazonnée de 27 p. 100.

Il apparaît ainsi que le quart de la réserve hydrique globale du sol des lysimètres cultivés est drainé annuellement, il faudrait donc 4 ans pour renouveler cette réserve.

\section{F. Evapotranspiration des lysimètres et consommation d'eau des cultures}

Pour le système de culture expérimenté, blé-betterave, l'évapotranspiration moyenne en eau est de $495 \mathrm{~mm}$. Elle correspond à une production moyenne de matière sèche de $11564 \mathrm{~kg} / \mathrm{ha}(14904 \mathrm{~kg} / \mathrm{ha}$ pour la betterave sucrière et $8224 \mathrm{~kg} /$ ha pour le blé). La quantité d'eau nécessaire à la 
production d'1 $\mathrm{kg}$ de MS, généralement désignée sous le nom de coefficient de transpiration, serait alors de : 4281 . Pour l'ensemble des cultures ce coefficient est de $550 \mathrm{l}$. à Versailles sur 18 années de mesures (BASTISSE, 1951) et de 553 1. à Quimper sur 9 années (COPPENET, 1969).

\section{G. Avantages et inconvénients des lysimètres en sols non remaniés}

Après 7 ans d'expérimentation, on peut donner un avis sur le fonctionnement des lysimètres, en matière de bilan de l'eau.

Un tel dispositif présente l'avantage de traduire exactement le fonctionnement des sols du milieu naturel, tout au moins pendant le drainage hivernal, avec possibilité d'investigation pour le fonctionnement des divers horizons, et ceci dès la $1^{\text {ère }}$ année de mise en service.

Par contre, en période de non-drainage, il y a un inconvénient dû au fait que la craie est coupée de son système poreux sous-jacent et que les mouvements capillaires ascendants sont supprimés. Enfin, au-dessus du plancher drainant, il se crée un état de saturation sur 20 à $25 \mathrm{~cm}$, dans le système poreux de la craie, modifiant légèrement les conditions de reprise de drainage et de réserve hydrique estivale.

Néanmoins, ce dispositif reste extrêmement intéressant pour une approche des bilans de l'eau du milieu naturel régional.

\section{CONCLUSION}

Après 7 années d'observations, les résultats obtenus sur les lysimètres en sol de craie non remanié, apportent des données nouvelles concernant : le drainage (durée, volume et temps de réponse aux précipitations), l'évaporation (en sol nu ou cultivé) et l'influence de la végétation. Les résultats essentiels sont les suivants :

\section{A. Durée du drainage}

Les lysimètres cultivés commencent à drainer normalement en décembre et la période de drainage dure 4 ou 5 mois. Elle s'arrête entre la mi-avril et la mi-mai.

En sol nu, la reprise du drainage est avancée d'un mois et des reprises de courte durée sont fréquentes au cours du printemps et de l'été selon l'importance des pluies.

\section{B. Volume du drainage - Relations : Précipitations-Drai- nage}

En sols cultivés, le drainage moyen est de $160 \mathrm{~mm}$ et représente 61 p. 100 des pluies de l'hiver et 24 p. 100 des précipitations annuelles. Le drainage de ces lysimètres est moins important que celui du lysimètre en sol nu $(284 \mathrm{~mm})$.
Par rapport à la réserve hydrique globale $(630 \mathrm{~mm}$ sur $200 \mathrm{~cm}$ d'épaisseur), le pourcentage du drainage représente 25 p. 100 en case cultivée, 45 p. 100 en case nue et 27 p. 100 en case engazonnée. Sous culture et en sol nu, il a été possible d'établir une corrélation satisfaisante entre le drainage et les précipitations. Sous gazon, il n'existe pas de corrélation satisfaisante.

En conclusion, la durée et le volume du drainage dépendent des conditions climatiques et du mode d'occupation $d u$ sol.

\section{Temps de réponse du drainage aux précipitations en période de drainage}

Les faibles précipitations $(\leqslant 10 \mathrm{~mm}$ ) ne fournissent pas de réponse au drainage.

Des précipitations supérieures à $10 \mathrm{~mm}$ sont nécessaires pour provoquer une reprise du drainage. Le temps moyen de réponse du drainage des lysimètres est de $12 \mathrm{~h} . \mathrm{m}^{-1}$.

La réponse du drainage du lysimètre $5(150 \mathrm{~cm}$ de profondeur) est en général en avance d'un jour sur celle des autres lysimètres $(200 \mathrm{~cm}$ de profondeur).

\section{Evaporation}

L'évaporation (pluie-drainage) des lysimètres varie selon le mode d'exploitation du sol. En lysimètre nu, l'évaporation est faible : $371 \mathrm{~mm}$; cette évaporation varie peu d'un dispositif lysimétrique à un autre et dépend peu du type de sol. En lysimètres cultivés, l'évapotranspiration est de $495 \mathrm{~mm}$ pour le système betterave-blé.

A partir de la production annuelle de matière sèche des cultures des lysimètres et de l'évapotranspiration globale (pluie-drainage), la quantité d'eau nécessaire à la production d'1 kg de MS est calculée ; le coefficient de transpiration serait de $428 \mathrm{l}$.

\section{E. Fonctionnement général des lysimètres}

Bien que la durée de fonctionnement des lysimètres soit encore faible, on constate une certaine similitude des résultats avec la Station de Versailles. Ceci confirme bien que le drainage des sols reste bien un fait d'ordre climatique, latitudinal, pour des sols de caractéristiques physiques voisines (profondeur, perméabilité).

On doit noter cependant que, pour les sols de craie où il existe un équilibre hydrique permanent avec le substrat, le lysimètre avec plancher drainant, modifie le fonctionnement hydrique en période de non-drainage. Néanmoins, un tel dispositif reste l'unique moyen d'une approche de bilan de l'eau des sols.

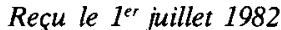
Accepté le 6 mai 1983.

\section{RÉFÉRENCES BIBLIOGRAPHIQUES}

Ballif J. L., 1978. Porosité de la craie. Appréciation de la taille et de la répartition des pores. Ann. agron., 29 (2), 123-131.

Ballif J. L., 1980. Caractères et réserves hydriques des sols sur craie et sur graveluche en Champagne. Ann. agron., 31 (4), 473-485.

Ballif J. L., 1981. Comportement hydrique d'une rendzine brune sur craie sous l'influence de la sécheresse. C. R. Acad. Agric. Fr., t. $67,395-403$.
Ballif J. L., Muller C., 1981. Azote minéral en sol de craie. I Incidences des conditions climatiques et du mode d'exploitation sur les pertes mesurées en lysimètres. Reims 7-10 juillet, Colloque humus-azote. A.I.S.S.-A.F.E.S. - Commissions III et IV, 218-225. Ballif J. L., Vachier P., 1981. Méthodes d'étude de la porosité et hypothèse sur le fonctionnement hydrique de la craie. Journ. Sci. du Groupe Français d'Humidimétrie Neutronique, Avignon, 24-25 novembre. 
Bastisse E. M., 1951. Dix-huit années d'études lysimétriques appliquées à l'agronomie. Premier mémoire, Ann. agron., série A, 721781.

Coppenet M., 1969. Résultats de douze années d'observations lysimétriques à Quimper (1954-1965). Ann. agron., 20 (2), 111-143. Dejou J., Morizet J., 1977. Etude comparative de la dynamique du drainage et de la composition des eaux de percolation dans les sols granitiques et basaltiques du Massif central. Résultats de 15 ans d'observation en cases de végétation dans les conditions climatiques du bassin limagnais. Ann. agron., 28 (4), 335-359.

Durand R., 1978. La pédogenèse en pays de craie dans le nord-est de la France. Thèse Sci. Univ. L. Pasteur, Strasbourg, 198 p.

Dutil P., 1981. Réalisation d'un dispositif de lysimétrie pour l'étude de la migration des solutés en sols non remaniés. Colloque humusazote, Reims 7-10 juillet - A.I.S.S.-A.F.E.S. - Commissions III et IV, 211-216.

Dutil P., Ballif J. L., 1968. Sur la présence fréquente en Champagne crayeuse de rendzines développées sur paléosols cryoturbés. Sci. Sol, 2, 79-92.
Dutil P., Muller J. C., 1979. L'épandage des eaux résiduaires des industries agricoles en Champagne crayeuse. C. R. Acad. Agric. Fr., 65, 989-1005.

Juste C., Tauzin J., Dureau P., Courpron C., 1982. Exportation des éléments fertilisants par lessivage en sol sableux des Landes de Gascogne. Résultats de 8 années d'observations en cases lysimétriques. Agronomie, 2 (1), 91-98.

Magniant D., Dutil P., 1971. Drainage accéléré en phase de dégel. Résultats d'une expérience sur modèle. C. R. Acad. Agric. Fr., 57, 61-67.

Muller J., 1977. Pouvoir épurateur du sol : cas de l'épandage des eaux résiduaires de féculerie. Etude en lysimètre en sol de craie non remanié. Sci. Sol, 3, 167-182.

Turc L., 1954. Le bilan de l'eau des sols: relations entre les précipitations, l'évaporation et l'écoulement. Ann. agron., série A, 491-595. 